(a) Catalase activity was measured by the method of Bonnichsen, Chance and Theorell ${ }^{6}$. All three substances depressed catalase activity per se, but protected the enzyme against radiation. At low radiation doses failure to take account of this depressing effect can lead to the apparent result of an increase of the radiation effect.

(b) Paper electrophoresis of unirradiated catalase showed a single spot, whereas irradiated catalase gave a comet-shaped streak. When irradiation took place in the presence of cysteine or glutathione, the subsequent electrophoretic pattern was the same as that of the unirradiated solution; that is, one spot appeared in the same position as unirradiated catalase. Protection occurred with cystine too, although to a lesser degree due to its low solubility.

(c) The chromatographic behaviour of catalase changed markedly after irradiation. This change did not occur when cysteine or glutathione was present during irradiation, and was not so pronounced when cystine was used.

(d) Catalase shows two maxima in the ultra-violet, at $275 \mathrm{~m} \mu$ and $405 \mathrm{~m} \mu$. In the present instance, $D_{405} / D_{275}=0.685$. After irradiation the absorption increased at $275 \mathrm{~m} \mu$ and decreased at $405 \mathrm{~m} \mu$, so that $D_{405} / D_{275}=0.226$. When cysteine or glutathione was present during irradiation, the absorption did not change.

Thus catalase is protected against $\gamma$-rays and highenergy electrons by cysteine, cystine and glutathione. It is noteworthy that the oxidized form of cysteine, namely, cystine, is nevertheless able to compete for the radicals produced by radiation.

This work will be reported in detail elsewhere. W. M. Dale

Department of Biochemistry, C. Russell

Christie Hospital and Holt Radium Institute, Manchester 20. Sept. 17.

${ }^{1}$ Forssberg, A., Nature, 159, 308 (1947).

2 Patt, H. M., Smith D. E., Tyree, E. B., and Straube, R. L., Proc. Soc. Exp. Biol. Med., 73 , 18 (1950).

${ }^{3}$ Chapman, W. H., and Cronkite, E. P., Proc. Soc. Exp. Biol. Med.. 75, 318 (1950).

- Kepp, R. K., and Michel, K. F., Strahlentherapie, 92, 416 (1953).

${ }^{5}$ Holmes, B., Nature, 165, 266 (1950).

- Bonnichsen, R. K., Chance, B., and Theorell, H., Acta Chem. Scand. 1, 685 (1947).

\section{Radiation Dosimetry of Rubidium-86}

INTEREST has recently arisen in the possibility of using rubidium-86 as a tracer for potassium in biological studies where the short half-life of potassium42 presents difficulties ${ }^{1}$. In order that an assessment may be made of the radiation dosage arising from the use of this isotope, the $\gamma$-ray output and mean $\beta$-ray energy have been measured. Rubidium-86 decays with the emission of a $1.80 \mathrm{MeV}, \beta$-ray or by a 0.72 $\mathrm{MeV}$. $\beta$-ray followed by a $1.08 \mathrm{MeV} . \gamma$-ray ${ }^{2}$. The results reported here indicate that the $\gamma$-ray occurs in about 8.4 per cent of disintegrations.

The material produced by neutron irradiation of the purest rubidium chloride at prosent available contains considerable activity from casium-134. A sample of the irradiated material was therefore purified by seven recrystallizations of the acid tartrate, after which only about 0.05 per cent of the disintegrations were due to eæsium-134 (as judged by the subsequent increase in the ratio of $\gamma$ - to $\beta$-ray activity over a period of three months). The disintegration-rate of an aliquot of this material was determined by $4 \pi$-ray counting, a method which has been found satisfactory for emitters of relatively high-energy $\beta$-rays ${ }^{3}$.

The $\gamma$-ray dose-rate was measured by comparison against a radium standard, using a graphite ionization chamber, and assuming that $\mathrm{I} \mathrm{mgm}$. of radium, screened by $0.5 \mathrm{~mm}$. of platinum gives $8.3 \mathrm{r} . / \mathrm{hr}$. at $1 \mathrm{~cm}$. The emission of $\beta$-ray energy was measured by the method of Gray ${ }^{4}$, in which the ionization produced in a chamber lined with agar containing a known concentration of the active material is compared with the ionization produced by a standard radium source at known distance. The ionization measurement was related to energy absorption on the assumption that $32.5 \mathrm{eV}$. is required for the formation of an ion-pair in air. It was found that the average energy emitted as $\beta$-rays per disintegration of rubidium-86 is $0.66 \mathrm{MeV}$., and that the $\gamma$-ray dose-rate at $1 \mathrm{~cm}$. from $1 \mathrm{mc}$. is $0.525 \mathrm{r} . / \mathrm{hr}$. An appreciable fraction of the $\gamma$-ray effect is due to bremsstrahlung. Since these radiations fall largely in a region where the sensitivity of the chamber changes rapidly with quantum energy, it is not possible to calculate the contribution, but its magnitude can be inferred from the figure $0.025 \mathrm{r} . / \mathrm{hr}$. at $1 \mathrm{~cm}$. obtained from 1 mc. of phosphorus-32 under similar conditions.

In view of the closeness of the maximum energies of the $\beta$-rays of these two isotopes, it is reasonable to assume that the bremsstrahlung effect is proportional to the square of the maximum energy, when, allowing for the presence of the low-energy branch, the bremsstrahlung of rubidium-86 would give $0.027 \mathrm{r}$. $/ \mathrm{hr}$. mc. at $1 \mathrm{~cm}$. The $1.08 \mathrm{MeV}$. $\gamma$-ray therefore gives $0.50 \mathrm{r} . / \mathrm{hr}$. per mc. at $1 \mathrm{~cm}$., a figure which leads to the conclusion that the $\gamma$-ray is emitted in only 8.4 per cent of disintegrations. Accepting this figure for the branching ratio, and allowing for the forbidden shape of the $\beta$-ray spectrum ${ }^{5}$, the calculated value of the mean $\beta$-ray energy is $0.70 \mathrm{MeV}$. The discrepancy between this figure and the experimental results is scarcely greater than the uncertainty of the value assumed for the energy of formation of an ion-pair.

A regression line calculated from seventeen observations over a period of 79 days gave a figure of $18.74 \pm 0.026$ days for the radioactive half-life of a sample of rubidium- 86 . This reduces to $18 \cdot 66$ days when allowance is made for the 0.05 per cent cæsium-134 initially present.

We are indebted to the Medical Research Council for the loan of the equipment used in the ionization measurements.

\section{E. W. EMERY}

Department of Medicine,

University, Manchester.

Postgraduate Medical School, London, W.12.

\section{J. E. S. BradLEY} London, W.12.

Physics Department,

Guy's Hospital Medical School, London, S.E.1.

Nov. 1.

${ }^{1}$ Love, W. D., Romney, R. B., and Burch, G. E., Cireulation Res., 2, 112 (1954).

'Hollander, J. M., Pearlman, I., and Seaborg, G. T., Rev. Mod. Phys., 25, 469 (1953).

${ }^{3}$ Perry, W. E., "Standardisation of Radioactive Isotopes" (National Physical Laboratory, 1953).

${ }^{4}$ Gray, L. H., Brit. J. Radiol., 22, 667 (1949).

${ }^{8}$ Wu, C. S., Rev. Mod. Phys., 22, 386 (1950). 